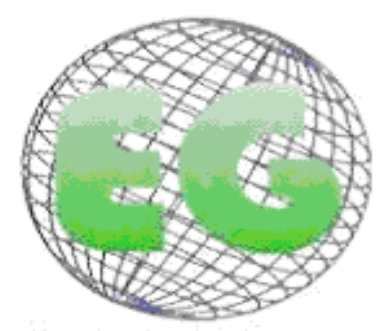

ISSN 1695-6141 N 25
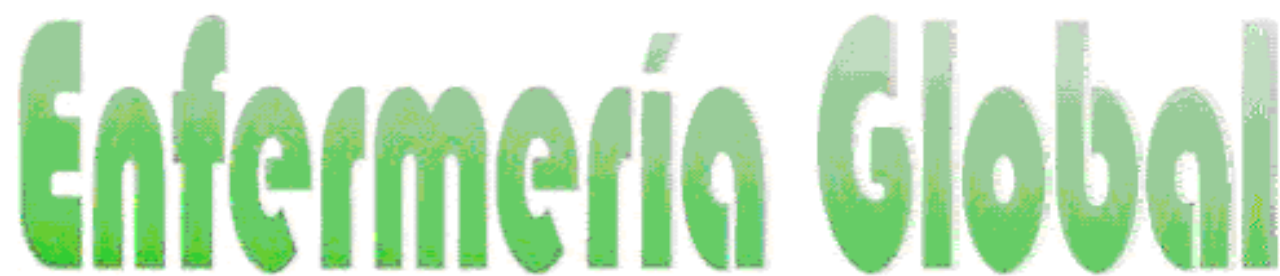

Revista electrónica trimestral de Enfermería

Enero 2012

www.um.es/egloball

\title{
Enfermeras de Atención Primaria opinan, analizan y proponen mejoras en el manejo de la adherencia farmacológica
}

Primary care nurses think, analyze and propose improvements ion pharmacological adherence

\author{
*Rodríguez García, MJ, **Del Castillo Arévalo, F. \\ *Centro de Salud de Infiesto.**Centro de Salud de Contrueces. Gijón. Asturias. \\ Palabras clave: adhesión a la medicación; investigación cualitativa; Atención Primaria \\ Keywords: pharmacological adherence, qualitative study, primary care
}

\section{RESUMEN}

Objetivo: Conocer cómo se percibe y aborda la adherencia farmacológica por enfermeras de Atención Primaria e identificar áreas de mejora.

Método: Estudio cualitativo mediante grupos focales y entrevistas en profundidad realizado en mayo y junio de 2009 en el Área Sanitaria V de Asturias. Audiograbadas y posteriormente transcritas para análisis de contenido.

Resultados: Se exponen los relativos a cómo valoran e intervienen sobre la adherencia farmacológica, las dificultades que encuentran y propuestas de mejora. Estiman que es un problema que debe ser tratado por médicos y enfermeras. Las dificultades que perciben son: el deficiente registro de los apartados de la receta, la escasa revalidación de la prescripción por los médicos; la ausencia de protocolos de registro de la actividad, y la insuficiente formación en farmacología por parte de las enfermeras. Rechazan el sistema de indicadores utilizados por el Sistema de Salud de la Comunidad por no valorar el quehacer cotidiano de las enfermeras. Proponen la mejora de los registros, formación en farmacología y metodología enfermera, el desarrollo de protocolos de valoración de la adherencia en el Área Sanitaria, y la implantación de programas pluridisciplinares.

Conclusiones. Las enfermeras otorgan gran importancia al problema del incumplimiento farmacológico, dedicándole tiempo y esfuerzos. Las dificultades que señalan restan efectividad a las intervenciones que realizan. Se necesita formación para un mejor manejo de este problema y la participación pluriprofesional (médicos, enfermeras, farmacéuticos, trabajadoras sociales, psicólogos clínicos y responsables del ámbito gerencial), pacientes y familiares para poner en práctica estrategias que optimicen los resultados.

\section{ABSTRACT}

Aim: To find out how primary care nurses perceive and handle pharmacological non-adherence and to identify areas for improvement. 
Methods: A qualitative study including focal groups and in-depth interviews was carried out in May-June 2009 in the Sanitary Area $V$ of Asturias, Spain. Interviews were recorded and transcribed in order to analyze the contents.

Results: Data on how nurses assess and handle pharmacological adherence are presented, as well as the difficulties they face and proposals for improvement. They believe this problem should be taken care of by both practitioners and nurses. The difficulties they find are: prescription fields deficiently recorded; the sparse revalidation of prescriptions by general practitioners; the lack of activity recording protocols; and the insufficient training on pharmacology nurses have. They reject the marker system used by the community Healthcare System because the daily routine of the nurses is not appreciated. They propose improving the records, the availability of pharmacology training and nursing training, the development of adherence assessment protocols in the Sanitary Area and the implementation of multidisciplinary programs.

Conclusions: Nurses give great relevance to the pharmacological non-adherence issue, and they devote time and effort to it. The impediments they highlight reduce the effectiveness of the interventions carried out. Training is necessary for a better handling of this issue, as well as the joint multidisciplinary participation of practitioners, nurses, pharmacists, social workers, clinical psychologists and managers, patients and relatives so as to implement strategies which may optimize the outcomes.

\section{INTRODUCCIÓN}

La OMS define la adherencia terapéutica como el grado en que el comportamiento de una persona - tomar el medicamento, seguir un régimen alimentario y ejecutar cambios del modo de vida- se corresponde con las recomendaciones acordadas de un prestador de asistencia sanitaria $^{1}$

El documento de la OMS recoge que la mala adherencia a los tratamientos crónicos es un problema mundial de alarmante magnitud, estimando el cumplimiento de los tratamientos a largo plazo en un $50 \%$ en los países occidentales.

Según diversos estudios, las consecuencias prácticas más destacables derivadas del incumplimientos son: causa principal de fallo terapéutico, aumento del riesgo de complicaciones y hospitalizaciones e incremento de los costes sanitarios al financiar fármacos que no se toman o se hace de forma inapropiada ${ }^{2,3,4,5,6,7}$

La falta de adherencia farmacológica es un fenómeno multifactorial, con un amplio abanico de factores determinantes que actúan de forma interrelacionada entre ellos ${ }^{3}$. Según el citado informe de la OMS, estos factores están relacionados con: los profesionales, los sistemas de atención sanitaria, el tratamiento, el paciente y la enfermedad, y socioeconómicos. Lo que añade gravedad al fenómeno es que el incumplimiento pasa desapercibido para el personal sanitario.

Algunos autores han calculado que la inversión en programas de mejora de la adherencia de tratamientos antihipertensivos produciría una disminución significativa de la discapacidad, incluso de la mortalidad, mayor que la que produciría una inversión de la misma magnitud en la detección y tratamiento de casos nuevos ${ }^{4}$. En nuestro país existen diversos programas de apoyo a pacientes polimedicados, todos ellos con un abordaje multidisciplinar y con la intención de realizar aproximaciones al problema de forma integral ${ }^{8 .}$

Las enfermeras por su proximidad al paciente, pueden contribuir eficazmente a disminuir los errores en la medicación y la falta de adherencia medicamentosa. Este problema es objeto de su atención como prueba el hecho de que el cumplimiento de indicaciones terapéuticas aparece entre los diagnósticos enfermeros más utilizados ${ }^{9}$ o priorizados por importancia percibida ${ }^{10}$ en Atención Primaria. 
El objetivo de este estudio es conocer cómo se aborda y percibe la adherencia farmacológica por las enfermeras de Atención Primaria en el Área sanitaria V de Asturias e identificar campos de mejora.

\section{MÉTODOS}

Estudio cualitativo mediante grupos focales y entrevistas en profundidad, realizado en mayo y junio de 2009 en el Área Sanitaria V de Asturias. Esta Área tiene asignada una población de 302.000 habitantes distribuida en 15 Centros de Salud urbanos y 5 Centros periféricos, de ellos 4 semiurbanos

Se convocaron tres grupos de 9 enfermeras cada uno. La segmentación de los grupos se realizó según el tiempo trabajado en Atención Primaria (AP): menos de 2 años, más de 10 años el segundo, y un tercer grupo formado por integrantes de la Comisión de Cuidados del Área o que desempeñan funciones de responsable de enfermería en el centro de salud. El punto de corte de menos de 2 años en AP viene dado porque es el periodo de tiempo transcurrido desde el último concurso de traslados.

Se contactó telefónicamente con las enfermeras para invitarlas a participar y se les envió una carta explicativa del proyecto recordando la fecha y lugar de la convocatoria, adjuntando la autorización de la Dirección de Enfermería.

En los grupos, que contaron con la asistencia del $100 \%$ de las personas invitadas, se llevaron a cabo entrevistas semiestructuradas. La duración media fue de 1h, 45 minutos.

Los procedimientos utilizados para garantizar el rigor del estudio son: la descripción detallada de las fases de la investigación (del muestreo y del análisis), y la triangulación, realizándose dos entrevistas en profundidad para obtener una visión más amplia y profunda del objeto de estudio.

Las entrevistas en profundidad se realizaron al director de enfermería del Área Sanitaria $\mathrm{V}$ y al coordinador del grupo de trabajo de diabetes de la Sociedad Española de Medicina Familiar y Comunitaria por el conocimiento y experiencia de ambos en el tema a estudiar. Se grabó en audio, previo consentimiento de los participantes para su posterior trascripción textual y análisis de contenido.

Las investigadoras actuaban una de moderadora y otra de observadora.

\section{RESULTADOS}

Los resultados se estructuran en cinco apartados: a) Consideraciones sobre la adherencia farmacológica, b) valoración y estrategias de prevención e intervención, c) incidencia de la organización asistencial en el manejo del cumplimiento, d) factores y situaciones que dificultan las actuaciones sobre la adherencia y e) propuestas de mejora.

\section{a) Consideraciones sobre la adherencia}

Existen discrepancias en la importancia que otorgan los participantes a la adherencia farmacológica. Las enfermeras, por lo general, prestan atención al cumplimiento de los pacientes con diagnóstico de HTA y diabetes, particularmente en personas mayores y sobre los fármacos prescritos para el tratamiento de esas dos enfermedades, pero la prevención, 
detección y seguimiento de la adherencia farmacológica no están integradas en la práctica clínica diaria para otras patologías crónicas y otro perfil de pacientes.

Consideran que el incumplimiento repercute en la falta de control del proceso y en la utilización ineficiente de los recursos, con el consiguiente aumento de los costes sanitarios. Algunos participantes opinan que se otorga mucha atención al control de los fármacos en detrimento de las recomendaciones sobre los estilos de vida, y que son estos cuidados de promoción o prevención los que singularizan el quehacer enfermero. Otros manifiestan que la revisión de los medicamentos que tiene prescritos el paciente y el seguimiento de cómo los toma, es una responsabilidad médica (Tabla 1).

\begin{tabular}{|l|l|}
\hline $\mathrm{G} 2 \mathrm{P}$ & $\begin{array}{l}\text { Creo que la adherencia podria manejaria mejor la } \\
\text { enfermera que el médico, lievar } \\
\text { mejor control de la medicación. }\end{array}$ \\
\hline $\mathrm{G} 2 \mathrm{P}$ & $\begin{array}{l}\text { Creo que estamos demasiado centrados en el } \\
\text { tratamiento farmacológico... estamos } \\
\text { dando más importancia a la medicación que a cambiar } \\
\text { hábitos. }\end{array}$ \\
\hline $\mathrm{G} 3 \mathrm{P}$ & $\begin{array}{l}\text { Muchas veces las enfemeras estamos lienando } \\
\text { huecos de otros profesionales, que } \\
\text { no lo hacen o lo hacen a medias, y a veces queriendo } \\
\text { tapar esos huecos no } \\
\text { hacemos lo fundamental, o lo nuestro, que es más, el } \\
\text { dar información, el Plan de } \\
\text { Cuidados. }\end{array}$ \\
\hline $\mathrm{G} 3 \mathrm{P}$ & $\begin{array}{l}\text { La adhesión al tratamiento es tan importante que } \\
\text { deberiamos todos incidir en ello; } \\
\text { como colectivo y sobre todo porque es calidad } \\
\text { asistencialy la seguridad que } \\
\text { necesita el paciente. }\end{array}$ \\
\hline $\mathrm{G} 1 \mathrm{P}$ & $\begin{array}{l}\text { No estamas solos con la medicación, la prescribe otra } \\
\text { persona, la pauta otra persona, } \\
\text { nosotros velamos por la seguridad del paciente y } \\
\text { veremas como lo tomay si lo toma, } \\
\text { pero la responsabilidad no es solo de la enfermera, } \\
\text { hay otros profesionales que tienen } \\
\text { esa misma responsabilidady que puede ser mayor }\end{array}$ \\
\hline
\end{tabular}

Nota: G corresponde al grupo y $P$ a la posición del participante dentro del grupo.

La mayoría entiende que mejorar el cumplimiento farmacológico de los pacientes es una responsabilidad compartida de médicos y enfermeras y lo argumentan diciendo que una atención integral al paciente, supone resolver aquellos problemas que detecte la enfermera y que es competente para tratar, y el manejo de la medicación es una intervención esencial del cuidado que se presta a los pacientes con enfermedades crónicas.

\section{b) Valoración y estrategias de prevención e intervención}

Para efectuar la valoración de la adherencia farmacológica, las enfermeras formulan preguntas directas, y de fácil respuesta, del tipo: ¿" se le olvida a Ud. tomar alguna vez las pastillas"? Otros métodos utilizados son el juicio clínico y la falta de control del proceso.

Manifiestan tener dificultades sobre a quién etiquetar de "incumplidor". Hay quienes consideran que el buen control de la enfermedad es un indicador de buen cumplimiento. 
Existen diferencias acerca de en qué lugar de la historia registrar la valoración de la adherencia, no obstante, la mayoría dice realizarlo en una carpeta de seguimiento de las patologías crónicas disponible en OMI-AP (historia clínica informatizada de Atención Primaria). Una de las quejas que exponen es que la opción de este ítem es dicotómica. Echan de menos un protocolo específico que facilite efectuar esta actividad.

Algunas enfermeras utilizan las taxonomías enfermeras en el manejo de la adherencia farmacológica. El tercer grupo hizo más hincapié en las dificultades y dudas tanto a la hora de elaborar un plan de cuidados (diagnóstico, resultados, indicadores, intervenciones y actividades) como de registrarlo en OMI. Los tres grupos manifiestaron que el uso de la metodología enfermera les resulta complejo y esta es poco utilizada.

Varias personas ponen de manifiesto la necesidad de registrar al menos con una etiqueta NIC (Clasificación de Intervenciones Enfermeras) la actividad que realizan sobre la adherencia farmacológica.

Prevención: En la entrevista médica, se declara que para evitar el incumplimiento, primero hay que convencer a los pacientes de la necesidad del tratamiento, de los beneficios que de él se derivan a largo plazo, explicarles los objetivos del tratamiento e implicarles en el cuidado de su salud (Tabla 2).

Tabla 2.- Intervenciones realizadas para prevenir y mejorar la adherencia farmacológica ( algunas de ellas son utilizadas indistintamente en ambas situaciones)

\begin{tabular}{|l|l|}
\hline Prevención & Responsabilizar al paciente e implicarle \\
en su autocuidado & Intimidación (inculcar miedo) \\
& Información verbal y escrita sobre la \\
enfermedad y su tratamiento & Preguntar sobre la adherencia en cada \\
& visita de seguimiento de la/s patología/s \\
Implicar a la familia, cuidadores y \\
allegados \\
Citar a consultas de seguimiento para \\
control de medicación \\
\hline Mejora y refuerzo & Derivar a la trabajadora social \\
& Recomendar sistemas personalizados \\
& de dosificación \\
& Reforzar la autoeficacia \\
& Potenciar la autoestima \\
& Elogiar y felicitar \\
\hline
\end{tabular}

Las enfermeras coinciden en que aceptar el diagnóstico es esencial para seguir adecuadamente el tratamiento. 
Detectado el incumplimiento, las intervenciones que realizan se centran en aumentar los conocimientos del paciente para que mejore su cumplimiento (Tabla 2).

En los casos de personas mayores que viven solas, con deficiencias cognitivas y merma de las capacidades manuales, tratan de implicar a su familia y si esto no fuera posible, lo ponen en conocimiento de la trabajadora social.

Si el cumplimiento es adecuado, refuerzan al paciente, asocian los datos clínicos positivos con el esfuerzo realizado, le felicitan y animan a seguir para garantizar que se repita y mantenga esa conducta.

Algunos de los sentimientos que la conducta no adherente desencadena en los informantes son la impotencia por los insuficientes resultados percibidos a pesar del tiempo y esfuerzo invertido, el enfado ante la falta de colaboración y compromiso del paciente y la empatía, que verbalizan con expresiones de ofrecerle ayuda, apoyo y comprensión.

\section{c) Incidencia de la organización asistencial en el manejo del cumplimiento}

En los grupos se discutió sobre el contenido de las consultas de seguimiento de las patologías crónicas. Hay partidarios de reducir las actividades técnicas y ser más proactivos en la revisión de la medicación y más concretamente de las personas mayores polimedicadas.

En la entrevista médica se sostiene que el papel de la enfermera es fundamental en la prevención, detección y seguimiento de la adherencia, pero que esta actividad no se realiza sistemáticamente, solo con pacientes muy concretos. Considera que se tiene interiorizada la importancia del estilo de vida, pero no las cuestiones relativas al tratamiento farmacológico.

En cuanto a la coordinación entre los miembros del equipo, las enfermeras dicen remitir el paciente al médico en los casos siguientes: la falta de control de la enfermedad, la duplicidad de fármacos, la toma de nuevos medicamentos que no aparecen en la historia, la solicitud por el paciente de la sustitución de un medicamento comercial por un genérico por motivos económicos y la presencia de efectos adversos. No dejan constancia escrita de la solicitud de esa interconsulta. Manifiestan que los médicos solicitan la intervención enfermera para el control de las cifras de presión arterial y/o glucemia después de un cambio del tratamiento, para iniciar medidas higiénico-dietéticas y en algún caso para averiguar qué fármacos toma el paciente cuando sospecha que no ha sido sincero con él.

Los aspectos sociales que pueden influir en la adherencia son abordados someramente en los grupos. El recurso que utilizan las enfermeras ante la problemática social es la derivación a la Trabajadora Social del Centro de Salud. En los centros que no disponen de este profesional asumen alguna de sus actividades, reconociendo que no es una labor propia, pero que no hacerlo repercutiría negativamente en la salud de la persona.

Sobre la coordinación Atención Primaria-Servicios Sociales, el director de enfermería y el médico coinciden en que no se ha avanzado nada en los últimos años e incluso se puede haber retrocedido.

Las enfermeras expresan malestar por los indicadores de evaluación de su actividad seleccionados por la Gerencia, entre los que no se encuentran las intervenciones que realizan para mejorar la adherencia farmacológica. Se preguntan si han de valorarse solo los datos cuantitativos o habría que incorporar también aquellas actuaciones de relevancia 
reconocida en la salud de los pacientes como es el seguimiento farmacológico. Reclaman que se visibilice su trabajo diario que desempeñan en la mejora del cumplimiento farmacológico, y su contribución en los resultados en salud obtenidos (Tabla 3).

Tabla 3.- Opiniones sobre la organización asistencial

\begin{tabular}{|l|l|}
\hline G3P3 & $\begin{array}{l}\text { A mi lo que me lieva los demonios es que la Hbat es el único indicador que } \\
\text { nos dice si está bien controlado el paciente; pero algo habré hecho yo } \\
\text { para que esa Hbat haya bajado de } 8 \text { a } 6 \text {; pues mi trabajo no viene en el } 1 S 1 \mathrm{~S}^{*}\end{array}$ \\
\hline G2P2 & $\begin{array}{l}\text { Se podria trabajar más, pero en este momento hay un desencanto por una } \\
\text { serie de cosas. A lo mejor se necesitarian más incentivos y valoración; } \\
\text { no tienen que ser incentivos económicos pero si la valoración de que } \\
\text { estas haciendo un trabajo, porque vemos que estamas trabajando más } \\
\text { y no aparecemas en ningún lado, solo aparecemos para mal. }\end{array}$ \\
& $\begin{array}{l}\text { El probiema mas importante es la falta de motivación, tu no puedes llegar a } \\
\text { estas aluras y limitarte a mirar la tensión porque eso ya está resuelto, } \\
\text { la importancia de la consulta no es tomar la tensión, la importancia es } \\
\text { el tratamiento no farmacológico, y más importante que tomar la TA es } \\
\text { saber como toma la medicación. }\end{array}$ \\
\hline G1P5 & $\begin{array}{l}\text { Yo estoy trabajando con esa educación de cómo debe tomar la medicación } \\
\text { un tiempo y en la historia solo hago que poner un punto. Si estoy } \\
\text { haciendo una educación de cómo tiene que fomar esa medicación, eso } \\
\text { tiene que constar ahi, alto y claro. }\end{array}$
\end{tabular}

*ISIS: Indicador sintético de infomación sanitaria para medir la productividad profesional.

Nota: $G$ corresponde al grupo y $P$ a la posición del participante dentro del grupo.

\section{d) Factores y situaciones que dificultan las actuaciones sobre la adherencia}

Una de las dificultades más reseñadas y que suscitan mayor unanimidad entre las enfermeras fue la relativa al incompleto registro por los médicos de los apartados que aparecen en el protocolo "prescripción" en la historia informatizada del paciente, así como la falta de actualización de la medicación. Campos importantes como la posología o la duración del tratamiento aparecen frecuentemente sin rellenar. Otras quejas que exponen son: la presencia de medicamentos repetidos con distinto nombre comercial; fármacos en desuso sin haber formalizado el cierre de la prescripción, medicamentos asociados a otro problema de salud para el que fueron recetados o incluidos en el episodio de actividades preventivas (Tabla 4). Si no se corrigen esas deficiencias a las enfermeras les resulta muy laborioso, desalentador y de resultado incierto, supervisar cómo el paciente está llevando a cabo el tratamiento y detectar posibles problemas de incumplimiento. 


\begin{tabular}{|c|c|}
\hline $\begin{array}{l}\text { Información parcial y confusa acerca de los } \\
\text { fármacos prescritos }\end{array}$ & $\begin{array}{l}\text { - Datos frecuentes sin cumplimentar: la } \\
\text { posología y/o duración del tratamiento. } \\
\text { - Repetición de principios activos con distinto } \\
\text { nombre comercial. } \\
\text { - Falta de actualización de las prescripciones en } \\
\text { uso. } \\
\text { - Fármacos no asociados al episodio CIAP } \\
\text { correspondiente. }\end{array}$ \\
\hline $\begin{array}{l}\text { Discontinuidad entre Atención Primaria y } \\
\text { Especializada }\end{array}$ & $\begin{array}{l}\text {-Causa de errores de medicación: pauta, dosis, } \\
\text { duplicidad de fármacos... }\end{array}$ \\
\hline $\begin{array}{l}\text { Insuficientes conocimientos en farmacología } \\
\text { de las enfermeras }\end{array}$ & $\begin{array}{l}\text { - Correspondencia entre principio activo y } \\
\text { nombre comercial. } \\
\text { - Indicaciones, posología, interacciones, efectos } \\
\text { secundarios, foma de administración. } \\
\text { - Desconocimiento fármacos nuevos. } \\
\text { - Supresión ante determinadas pruebas }\end{array}$ \\
\hline $\begin{array}{l}\text { Falta de protocolo para la valoración y el } \\
\text { registro }\end{array}$ & $\begin{array}{l}\text { - Única referencia a la adherencia farmacológica } \\
\text { en la historia informatizada como variable } \\
\text { dicotómica (Si/No) } \\
\text { - Ausencia de criterios consensuados en el Área } \\
\text { Sanitaria para etiquetar el incumplimiento }\end{array}$ \\
\hline
\end{tabular}

El médico entrevistado manifiesta similares opiniones, admitiendo que el registro de la posología es, en general, deficiente.

Otra dificultad que exteriorizan, de forma más destacada en el grupo de enfermeras que trabaja en A.P. desde hace menos de 2 años, es el insuficiente conocimiento de los fármacos utilizados en las patologías más prevalentes. Dicen encontrar problemas para relacionar principios activos con nombres comerciales, identificar interacciones entre fármacos, desconocer los medicamentos de reciente comercialización, cómo se administran y sus efectos adversos, y en qué situaciones está recomendada la suspensión de ciertos fármacos ante determinadas pruebas diagnósticas.

En las dos entrevistas también se pone de manifiesto la conveniencia de mejorar los conocimientos farmacológicos de las enfermeras. El médico estima que este déficit puede explicar que algunos de estos profesionales consideren el seguimiento farmacológico como tarea de otros.

\section{e) Propuestas de mejora}

Se han agrupado en cuatro apartados: formación, sistema de registro, medidas psico-sociales y organización. Para facilitar su lectura se detallan en la Tabla 5. 
Tabla 5.- Propuestas para mejorar la adherencia farmacológica

\begin{tabular}{|l|l|}
\hline Formación & $\begin{array}{l}\text { - Aumentar conocimientos y habilidades en farmacología y metodología enfermera a } \\
\text { través de: } \\
\text {-Sesiones clínicas de enfermería. } \\
\text {-Sesiones con famacéutico de Area }\end{array}$ \\
\hline Sistema de registro & $\begin{array}{l}\text { - Usar etiquetas NIC que recojan las actividades realizadas } \\
\text { - Rellenar los campos-clave de las recetas por parte del prescriptor; asociar el } \\
\text { fármaco al episodio ClAP correspondiente y actualizar la medicación en uso e } \\
\text { inactivar el resto } \\
\text { - Elaborar un protocolo de valoración apropiado } \\
\text { - Incluir en el informe de continuidad de cuidados el tratamiento farmacológico del } \\
\text { paciente. } \\
\text { - Conseguir que la aplicación informática OMl-Planes sea más accesible e intuitiva } \\
\text { - Consensuar criterios para unificar los registros en la historia clínica }\end{array}$ \\
\hline Medidas psico-sociales & $\begin{array}{l}\text { - Mejorar la coordinación con los servicios sociales } \\
\text { - Organizar grupos de autoayuda de pacientes de edad media con patología crónica } \\
\text { - Mejorar los conocimientos y habilidades psico-sociales } \\
\text { - Colaborar con psicólogos clínicos } \\
\text { - Seleccionar población diana de atención preferente (polimedicados y edad media) }\end{array}$ \\
\hline Organización & $\begin{array}{l}\text { - Reconocer la adherencia por parte de la Gerencia como área de interés prioritario } \\
\text { - Seleccionar indicadores de actividad que visibilicen el trabajo enfermero } \\
\text { - Motivar- sensibilizar a los profesionales sobre la importancia de mejorar la } \\
\text { adherencia } \\
\text { - Elaborar un protocolo de Área que facilite el desempeño de esta actividad } \\
\text { Implicar a los farmacéuticos comunitarios en la prevención, detección y mejora del } \\
\text { cumplimiento. }\end{array}$ \\
\hline
\end{tabular}

\section{DISCUSIÓN}

En las consultas programadas de control de las patologías crónicas, las enfermeras efectúan actividades técnicas, informativas y de revisión del cumplimiento de los fármacos. El modelo que prevalece sigue estando basado en la enfermedad y en las actividades delegadas del diagnóstico y tratamiento médico más que en las necesidades del paciente ${ }^{11}$. Predomina la educación sanitaria estandarizada, la no aplicación del proceso enfermero de atención, y el control de la enfermedad, sobre la promoción de la salud y el autocuidado.

Este tipo de práctica tiende a fomentar comportamientos y actitudes pasivas y dependientes en los pacientes en lugar de promover una mayor implicación y autorresponsabilidad en el manejo efectivo de sus problemas de salud ${ }^{12,13}$.

Coincidiendo con la tesis mantenida por Morales Asencio et $\mathrm{al}^{14}$., algunos de los participantes perciben el control de la adherencia farmacológica como algo externo a ellos, propio de los médicos y que por lo tanto no se encuentra entre sus funciones habituales. Valoran el tiempo y esfuerzo dedicado a verificar el cumplimiento de los medicamentos de excesivo y una desviación del sentido de su ejercicio profesional.

Otras enfermeras conciben la adherencia de los medicamentos como un aspecto más del cuidado integral del paciente. 
Al indagar por cómo llevan a cabo la detección de la adherencia, se observa que aunque existe un amplio número de tests de valoración indirecta del cumplimiento terapéutico ${ }^{15}$, no han sido integrados como recurso clínico por las enfermeras. La ventaja que se atribuye al empleo de estos procedimientos es que mejoraría la identificación de los pacientes incumplidores.

Una dificultad que manifiestan las enfermeras es cuándo considerar incumplidor a un paciente. Dicen carecer de criterios claros que les facilite clasificarle en una u otra categoría de la variable dicotómica adherente- no adherente. Según la literatura, se considera paciente cumplidor si observa el tratamiento por encima del $75-80 \%$, o bien el intervalo entre el 80 $110 \%$ de la dosis prescrita ${ }^{2}$. No obstante, falta unanimidad en cuanto a la consideración del concepto, la variedad de formas que adopta el incumplimiento y a su cuantificación.

Las intervenciones mencionadas por las enfermeras para facilitar la adherencia se podrían clasificar en: 1) educativas, orientadas a proporcionar información (oral y escrita) y mejorar los conocimientos sobre la enfermedad, el tratamiento y la importancia de la adherencia; 2) conductuales: asociar la toma del fármaco con una actividad diaria habitual, pastilleros, consultas de revisión más frecuentes, reconocimiento explícito ante la consecución de objetivos y 3) de apoyo familiar y social. Hay bastante coincidencia en los estudios publicados $^{16,17}$ en afirmar que no existe ninguna intervención específica que haya demostrado ser efectiva en todos los pacientes y en recomendar la combinación de actuaciones ya que han obtenido resultados más positivos que las intervenciones aisladas. Además se considera necesario personalizar las actuaciones según los factores implicados en el incumplimiento y las características del paciente y su entorno ${ }^{18}$.

En este estudio no se indaga si las intervenciones se utilizan de forma aislada o conjunta, ni qué modalidades se combinan con más frecuencia.

En una revisión sistemática ${ }^{19}$ publicada en 2007, se evaluó el impacto de algunas intervenciones dirigidas a facilitar el compromiso de los pacientes en su autocuidado. A pesar de que la calidad de las revisiones estudiadas, según los autores, fue muy variable, de las iniciativas analizadas para mejorar la adherencia terapéutica la que demostró mayor efectividad fue la simplificación del tratamiento, encontrándose mejoras menos concluyentes para otras intervenciones que los autores agrupan en las categorías de educación al paciente, información y counselling.

En 1996 se llevó a cabo otra revisión ${ }^{20}$ sobre las intervenciones más efectivas para ayudar a los pacientes a cumplir los tratamientos farmacológicos y concluyó que para conseguir los máximos beneficios de los medicamentos, era preciso hallar fármacos curativos con el mínimo de efectos adversos y comprender mejor los factores implicados en la adherencia así como descubrir qué combinaciones de estrategias son más beneficiosas para los pacientes. Puesto que los tratamientos autoadministrados continúan aumentando, sugieren que la investigación en adherencia básica y aplicada podría proporcionar más beneficios sobre la salud que los fármacos mismos.

\section{CONCLUSIONES}

Este estudio pone de manifiesto:

1- La importancia otorgada por los informantes al problema de la falta de adherencia farmacológica. Las enfermeras centran la atención fundamentalmente en el cumplimiento de 
los fármacos prescritos para el control de la HTA y diabetes y en menor medida para otras patologías.

2- El seguimiento a los pacientes con patologías crónicas reproduce un modelo biomédico centrado en la enfermedad. Escasa implantación de una perspectiva cuidadora con enfoque integral de las necesidades de la persona.

3- La invisibilidad de la actividad de las enfermeras y su contribución a los resultados en salud se ve perpetuada por los indicadores seleccionados por la Administración Sanitaria para medir la actividad enfermera.

4- La necesidad de: a) cumplimentar las prescripciones por los médicos, b) crear un protocolo informático que facilite el registro de las diferentes actividades desarrolladas en la prevención y mejora de la adherencia farmacológica y c) aumentar la formación de las enfermeras en farmacología.

\section{BIBLIOGRAFÍA}

1- Sabate E. Adherencia a los tratamientos a largo plazo. Pruebas para la acción (Documento OMS traducido). Ginebra: Organización Mundial de la Salud 2004. Consulta: 5 Febrero 2009. Disponible en http:// www.paho.org/Spanish/AD/DPC/NC/adherencia-largoplazo.pdf

2- Palop Larrea V, Martínez Mir I. Adherencia al tratamiento en el paciente anciano. Información Terapéutica del Sistema Nacional de Salud 2004; 28: 113-120

3- García Díaz B. El cumplimiento terapéutico y los factores de influencia. Monografía 28. Año 2000. Consulta: 5 Febrero 2009. Disponible en : www.esteve.org.

4- Rodriguez Marín J. Cumplimiento terapéutico del paciente y seguridad clínica. Consulta: 12 Marzo $2009 . \quad$ Disponible en http://www.fundacionmhm.org/pdf/Mono8/Articulos/articulo7.pdf

5- Información Farmacoterapéutica de Euskadi . Medicación en el anciano. Vol 17, no 6 . 2009

6- Rigueira García A.I. Cumplimiento terapéutico:¿qué conocemos en España? Aten Primaria $2001 ; 27: 559-568$

7- Fernández Lisón L.C., Barón Franco B., Vázquez Domínguez B. Errores de medicación e incumplimiento terapéutico en ancianos polimedicados. Farm Hosp. 2006; 30(5): 280-283.

8- Villafaina Barroso A, Gavilán Moral E. Programas de apoyo al polimedicado II: Experiencias en el Sistema Nacional de Salud e internacionales desde la oficina de farmacia al ámbito hospitalario. Abordaje integral del paciente polimedicado, 2009. Consulta: 12 Marzo 2009. www.diariomedico.com /www.correofarmaceutico.com

9- Maña M, Fernández E, Veledo P, García P, González A, Soto L, Mesas A. Enfermeras centinelas en red. Consulta: 12 Marzo 2009.

http://www.enfermeriacomunitaria.org/documentos/bec/bec 27/centinela.pdf

10- Brito Brito PR. Diagnósticos enfermeros priorizados en atención primaria. Enferm. glob., Murcia, n. 16, jun. 2009. Consulta: 18 Agosto 2009.

Disponíble en <http://scielo.isciii.es/scielo.php?script=sci_arttext\&pid=S1695-

$61412009000200008 \&$ Ing $=$ pt\&nrm=iso $>$.

11- Análisis y propuestas de futuro para la mejora del trabajo enfermero en Atención Primaria. Jornadas FAECAP 2006.

12- Papel de Enfermería en Atención Primaria. Comisión Científico Técnico de Atención Primaria de la Comunidad de Madrid. Plan de mejora de atención primaria de la Comunidad de Madrid 2006-2009. Enero 2009 
13- Del Pino Casado R., Martínez Riera J.R. Estrategias para mejorar la visibilidad y accesibilidad de los cuidados enfermeros en Atención Primaria de salud. Rev Adm Sanit. 2007;5(2):311-37

14- Morales Asencio J.M., Martín Santos J.M., Contreras Fernández e., Morilla Herrera J.C. Prescripción de medicamentos y productos sanitarios por enfermeras comunitarias. Enferm Comun 2006; 2(1):8-16.

15- Rodríguez Chamorro M.A., García Jiménez E., Amariles P., Rodríguez Camorro A., Faus M.J. Revisión de tests de medición del cumplimiento terapéutico utilizados en la práctica clínica. Ate. Primaria 2008; 40(8): 413-7

16- Roter Debra L., Hall Judit A., Merisca R., Nordstrom B. Effectiveness of interventions to improve patient compliance: a meta-analysis. Med Care 1998. Vol 36(8) 1138-1161.

17- Medicines adherence. NICE clinical guideline 76. January 2009. Consulta: 12 Marzo 2009. Disponible en www.nice.org.uk.

18- Salvador Carulla J., Melgarejo Ochoa M. Cumplimiento Terapéutico. Ars Médica. 2002

19- Coulter A., Ellins J. Effectiveness of strategies for informing, educating, and involving patients. BMJ 2007;335;24-27.

20- Haynes B., Mckibbon K. A., Kanani R. Systematic review of randomised trials of interventions to assist patients to follow prescriptions for medications. The Lancet 1996; vol 348. August 10: 383-386 